\title{
What's the use of picture discrimination experiments?
}

\author{
Stephen E. G. Lea \\ University of Exeter
}

Keywords: picture-object equivalence, picture perception, categorization, visual discrimination

Why would we carry out experiments in which pigeons are trained to discriminate between sets of photographs of real-world objects? Weisman and Spetch (2010) suggest a number of possible reasons. Three that play a particularly important role in the discussion are:

(a) To discover whether birds can discriminate between the corresponding categories of real objects

(b) To discover the features of natural stimuli that elicit natural responses from birds

(c) "Mainly, what we want to know about is how birds represent objects" (Weisman \& Spetch, 2010, p. 27).

If we want to know whether it is useful to examine picture set discriminations, we need to ask which of these reasons lies behind the experiment in question. Of course, things are never as simple as that. Researchers are not always either clear or consistent about why we do experiments, and often more than one of these motivations may be detectable in the design and description of their research. Furthermore, those who read and cite research often attribute motives to the researchers that they did not in fact hold, or interpret results in ways that the original authors would not endorse. Nonetheless, the research techniques that are appropriate depend critically on which of these motivations are operative, and it follows that how we should assess the experiments and our results depends critically on which of these goals

Stephen E. G. Lea, School of Psychology, University of Exeter, United Kingdom. Send correspondence to s.e.g.lea@exeter.ac.uk. they are aiming at. In this commentary, I argue that the underlying motivation for most of the research that Weisman and Spetch (2010) discuss is (c) above, to investigate object representation. However, much of Weisman and Spetch's (2010) critique assumes that it is (a), to investigate the discriminability of the real objects. They build a damning case against the use of much of the published research to answer that question. But it is not clear that there is anyone in the dock - and in any case, it will be argued below that it is not improper to argue from the discriminability of pictures to the discriminability of the objects depicted, though the converse argument does indeed fail. Weisman and Spetch (2010) also argue strongly for the use of playback experiments, which are indeed the most usual and useful method for answering questions driven by (b) above, discovering the releasers of natural responses, but of limited use if what we are really interested in is object representation.

\section{What can picture set discrimination tell us about object set discrimination?}

Wesiman and Spetch (2010) argue that "knowledge about correspondence between pigeons' representations of pictures and of real objects is crucial to understanding the results of experimental tests using only pictures", and that seems uncontroversial. However, it is worth pausing to consider whether they are overstating the extent to which we do not already have the knowledge that they say is required.

The first source of such knowledge is logic. For any 
perceiver, pictures are nearly always highly impoverished representations of the corresponding real objects. Pictures are taken from a fixed perspective, rather than the variable one that an organism can obtain as it moves its eyes, head or body; they are in two dimensions rather than three; they are usually static; their representation of colour is imperfect (and this is true even if the picture is taken by a process designed for the eyes of the viewer rather than for those of some other species or genotype within the species); they are purely visual, instead of being complemented by other sensory channels; they cannot be manipulated; and they do not respond to the viewer, as the real object will if it is another animal. Relative to the real object, the representation is likely to be not just impoverished, but also distorted because a picture will not engender processes like size or colour constancy. However, it does not follow that picture discrimination is irrelevant to object discrimination. If an animal can discriminate between pictures of two sets of objects, it is almost inevitable that it will also be able to discriminate between the corresponding sets of objects because although making a picture impoverishes the stimuli, it is unlikely to add information to them that would be useful in the discrimination.

Furthermore, although it may distort the information in the stimuli, it is likely to do so in corresponding ways for all stimuli; for example, if there is discriminable colour information in the picture sets, it is likely to derive from discriminable colour information in the object sets, even if it is not quite the same information as with the real objects. Finally, if the individual pictures in a set can be discriminated, but nonetheless, the categories of pictures can also be discriminated, it is likely that the same would be true of the objects the pictures represent; although the information in the pictures is impoverished, it is likely that the same relative similarities between instances, within and between categories, will hold for the pictures as for the objects. In short, the fact that a bird can discriminate sets of pictures of objects is good evidence that it has sufficient perceptual and cognitive capacities to discriminate the corresponding sets of objects, and would in fact be able to discriminate them (and it may be a great deal easier to carry out the experiment with the pictures than the objects).

The converse, however, is not true - a failure to discriminate sets of pictures does not imply that the corresponding object sets could not be discriminated. This is why, for example, Ryan and Lea (1994), having found that pigeons could not discriminate between sets of photographs of other individual pigeons, did not conclude that pigeons could not discriminate between live individuals, but proceeded to test whether they could or not (and, not surprisingly, found that they could). Such results then set an agenda for further research to find out what the crucial information is that has been lost in the transformation from object to picture.

One particular kind of stimulus impoverishment gets a great deal of attention in Weisman and Spetch's (2010) critique, and that is the reduction of the (at least) tetrachromatic colour world of the typical bird to the trichromatic world of photographic processes adjusted to the normal human eye. While there is no doubt that this is an issue, its significance should not be overstated. There are a number of reasons for thinking that it is not as overwhelming as Weisman and Spetch argue.

First, as Weisman and Spetch (2010) indeed point out, humans are capable of coping well with pictures that do not map into the full richness of our colour vision. They point to the case of black and white photography and cinematography, but an even more extreme case is that of line drawing. Of course, monochrome pictures have colour information that is not so much wrong as missing; but the roughly $6 \%$ of human males who are anomalous trichromats do not find it impossible to recognize objects in ordinary colour photographs, which are taken with technology adapted to the modal set of cone absorption spectra, and must therefore give them wrong colour information. If humans can do this, there is no obvious reason why birds could not.

Secondly, however different the retinas of birds and humans are, nothing prevents us either from testing just how much distortion our existing colour technology is introducing, or from constructing pictures that will truly represent the coloration of the corresponding objects. It is an easy matter to record the spectrum of light coming off each point of a picture, and compare it with the spectrum coming from the corresponding point of an object; if the two are the same, then the picture will have the same colour effect as the object, no matter what cone systems the perceiver's retina contains. While the two are unlikely to be identical when pictures are produced by conventional human photographic technology, they may not be as different as we might think. And if they do tend to be very different, if we know what cone systems an animal has (and for pigeons we do know that: Govardovskii \& Zeuva, 1977; Bowmaker, Heath, Wilkie \& Hunt, 1997; Kawamura et al., 1999), we can in principle produce pictures that will have the same effect on those systems as the object would, just as conventional photographic technology does for humans. This is technologically challenging, but straightforward in principle; Endler's work on bower birds (e.g. Endler \& Day, 2006) shows how to go about it. 


\section{What can experiments with pictures tell us about the features of natural stimuli that elicit natural responses from birds?}

Weisman and Spetch (2010) make much of the possibilities of playback experiments. They derive them from the use of audio playback in research on birdsong, but their history is probably as long in the visual domain. The classical ethologists' experiments on releasing stimuli for species-typical fixed action patterns, which go back at least to Tinbergen and Kuenen (1939/1972), are simply playback experiments in the visual domain. The availability of cheap video technology has made it easier to do more elaborate kinds of visual playback (though with greater possibilities for errors due to the different characteristics of different species' visual systems) but it has not introduced any new principle.

Visual playback - that is, the presentation of pictures or videos of objects - is ideally suited to addressing the question of what features of naturally occurring visual stimuli elicit natural, unconditional responses from animals. The fact that the images used in such experiments are generally impoverished is in fact an advantage, because if we want to find out what the critical features for eliciting a natural response are, we are looking for the most impoverished stimulus that will do the job. In this context, Weisman and Spetch (2010) run into a tricky debate, when they try to distinguish between a situation where "natural responses to a pictured conspecific might possibly be elicited by some simple feature common to a video image and the real object" and "recognition of the picture as corresponding to the whole object" (p. 20). This comes close to asking questions that cannot be answered about the birds' subjective experience. They argue that "The discovery of relatively simple releasing stimuli is ... uninteresting in most studies of object recognition, because object recognition is rarely controlled by a single perceptual dimension". This argument is unconvincing. In the first place, as Tinbergen and Perdeck (1950) demonstrated, even simple fixed action patterns are generally released by multiple cues rather than a single one. In the second place, it may not be true that object recognition is rarely controlled by a single dimension, or at least a small set of dimensions; certainly when birds are trained to discriminate between complex two-dimensional patterns, they frequently do so using fewer dimensions than are available, fewer than would enable perfect performance, and fewer than humans use except when they are using verbalised rules (e.g. Lea, Wills \& Ryan, 2006; Wills, Lea, Leaver et al., 2009; Lea, Wills, Leaver et al., 2009).

\section{What can picture set discrimination tell us about how birds represent objects?}

Playback experiments are only useful when the stimuli concerned elicit some kind of unconditional response. Nonetheless, they can be used to tell us something about object representation, particularly when animals have different responses to different members of an object set. The classic example is in studies of individual, kin or species discrimination, where different unconditional responses may be made to images of apparently similar objects (e.g. Bradshaw, 1992; Guzman \& Marin, 2008; Hansen, Johannessen \& Slagsvold, 2008), or an unconditional response may be habituated to exemplars of one set of images, and then dishabituation can be seen when an exemplar of a different category is presented.

For the most part, however, if we want to study how birds represent particular objects, we are forced into training them to make distinct responses to representations of different objects. Of course, it is possible to train object discriminations directly, as was done by Delius (1992). But if we want to explore how objects are represented, we usually need to use impoverished versions of objects, because once again we are in search of the minimal information needed to trigger the response that the bird has been trained to make to the object.

The majority of experiments that have as their goal an understanding of the representation of objects, however, are not concerned with particular objects, but rather with the process of representation in general. As such they take for granted a crucial feature of object perception, namely that different objects within the same category, which require the same response (whether that response is conditional or unconditional), are discriminably different. Object recognition, therefore, is a problem of category discrimination. The major motivation of most experiments on the discrimination of picture sets by pigeons has in fact been to gain an understanding of what sorts of categories are discriminated, and how it is done.

Weisman and Spetch (2010) recognize (p. 23) that in "research on basic sensation and perception, as in color perception, edge detection, depth perception, motion detection, pattern recognition and shape recognition" the use of pictures may be appropriate, because "the assumption that simple visual processes apply generally in the laboratory and in nature" is reasonable. What they miss is that the same is true in research on basic cognition. Researchers with an interest in object representation use discriminations between sets of pictures of natural objects because those sets are likely to have the same structure, in terms of relative similarities, as the sets of views of a particular natural object that the bird will experience in normal life. Indeed, they are very likely 
to offer a better model of the structure of such categories than the kinds of artificial category introduced by Lea and Harrison (1978). It is in this sense, and not because we expect that the birds will recognize the objects they represent, that they are more ecologically valid than abstract patterns.

\section{Conclusion}

Picture-object correspondence is an important subject in comparative visual cognition. We would all like to know whether a pigeon sees a colour slide as a representation of a person, or simply as an abstract coloured pattern. As Weisman and Spetch's (2010) review demonstrates, it is not an easy question to answer, and the recent efforts of researchers like Aust and Huber $(2006,2010)$ and Dittrich et al. (2010) are welcome and fascinating. No doubt researchers do sometimes slide from a demonstration that birds can discriminate sets of pictures to discussions that assume that the birds see the pictures as representations of the corresponding objects. But it does not follow that there are no interesting questions about avian visual cognition that can be addressed by experiments on picture discrimination without first resolving the picture-object correspondence problem for the pictures and subjects concerned. On the contrary, the majority of the published experiments that have used picture discriminations have been interested in questions about category discrimination to which the issue of picture-object correspondence is largely irrelevant.

\section{References}

Aust, U., Huber, L. (2006). Picture-object recognition in pigeons: Evidence of representational insight in a visual categorization task using a complementary information procedure. Journal of Experimental Psychology: Animal Behavior Processes, 32, 190-195. doi:10.1037/00977403.32.2.190 PMid:16634663

Aust, U., Huber, L. (2010). Representational insight in pigeons: comparing subjects with and without real-life experience. Animal Cognition, 13, 207-218. doi: $\underline{10.1007 /}$ s10071-009-0258-4 PMid:19593601

Bowmaker, J. K., Heath, L. A., Wilkie, S. E., Hunt, D. M. (1997). Visual pigments and oil droplets from six classes of photoreceptor in the retinas of birds. Vision Research, 37, 2183-2194. doi:10.1016/S0042-6989(97)00026-6

Bradshaw, R. H. (1992). Conspecific discrimination and social preference in the laying hen. Applied Animal Behaviour Science, 33, 69-75. doi:10.1016/S01681591(05)80086-3

Dittrich, L., Adam, R., Ünver, E., Günturkün, O. (2010). Pigeons identify individual humans but show no sign of recognizing them in photographs. Behavioural Processes, 83, 82-89. doi:10.1016/j.beproc.2009.10.006 PMid:19887102
Endler, J. A., Day, L. B. (2006). Ornament colour selection, visual contrast and the shape of colour preference functions in great bowerbirds, Chlamydera nuchalis. Animal Behaviour, 72, 1405-1416. doi:10.1016/j. anbehav.2006.05.005

Govardovskii, V. I., Zueva, L. V. (1977). Visual pigments of chicken and pigeon. Vision Research, 17, 537-543. doi:10.1016/0042-6989(77)90052-9

Guzman, D. A., Marin, R. H. (2008). Social reinstatement responses of meat-type chickens to familiar and unfamiliar conspecifics after exposure to an acute stressor. Applied Animal Behaviour Science, 110, 282-293. doi: 10.1016/j. applanim.2007.04.017

Hansen, B. T., Johannessen, L. E., Slagsvold, T. (2008). Imprinted species recognition lasts for life in free-living great tits and blue tits. Animal Behaviour, 75, 921-927. doi:10.1016/j.anbehav.2007.07.023

Kawamura, S., Blow, N. S., Yokoyama, S. (1999). Genetic analyses of visual pigments of the pigeon (Columba livia). Genetics, 153, 1839-1850. PMid:10581289 PMCid:1460878 http://www.genetics.org/cgi/content/ abstract/153/4/1839

Lea, S. E. G., Harrison, S. N. (1978). Discrimination of polymorphous stimulus sets by pigeons. Quarterly Journal of Experimental Psychology, 30, 521-537. doi: $10.1080 / 00335557843000106$

Lea, S. E. G., Wills, A. J., Ryan, C. M. E. (2006). Why are artificial polymorphous concepts so hard for birds to learn? Quarterly Journal of Experimental Psychology, 59, 251267. doi:10.1080/02724990544000031 PMid:16618633

Lea, S. E. G., Wills, A. J., Leaver, L. A., Ryan, C. M. E., Bryant, C. M. L., Millar, L. (2009). A comparative analysis of the categorization of multidimensional stimuli: II. Strategic information search in humans (Homo sapiens) but not in pigeons (Columba livia). Journal of Comparative Psychology, 123, 406-420. doi:10.1037/ a0016851 PMid:19929109

Tinbergen, N., Kuenen, D. J. (1972). The releasing and directing stimulus situations of the gaping response in young blackbirds and thrushes (Turdus m. merula $L$. and T. e. ericetorum Turton). In N. Tinbergen, The animal in its world (Vol. 2), 17-51. London: Allen \& Unwin. (Originally published, 1939).

Tinbergen, N., Perdeck, A. C. (1950). On the stimulus situation releasing the begging response in the newlyhatched herring gull chick (Larus argentatus Pont.). Behaviour, 3, 1-39. doi:10.1163/156853951X00197

Weisman, R., Spetch, M. (2010) Determining when birds perceive correspondence between pictures and objects: a critique. Comparative Cognition and Behavior Reviews, 5, 117-131. Retrieved from http://psyc.queensu.ca/ccbr/ index.html doi: 10.3819/ccbr.2010.50006

Wills, A. J., Lea, S. E. G., Leaver, L. A., et al. (2009). 
A comparative analysis of the categorization of multidimensional stimuli: I. Unidimensional classification does not necessarily imply analytic processing; evidence from pigeons (Columba livia), squirrels (Sciurus carolinensis) and humans (Homo sapiens). Journal of Comparative Psychology, 123, 391-405. doi:10.1037/ a0016216 PMid:19929108 